\title{
Understanding the impact of socioeconomic differences in breast cancer survival in England and Wales: avoidable deaths and potential gain in expectation of life.
}

M. J. Rutherford ${ }^{1, *}$, T. M-L. Andersson ${ }^{2}$, H. Møller ${ }^{3}$, P.C. Lambert ${ }^{1,2}$.

${ }^{1}$ Department of Health Sciences, University of Leicester, UK. LE1 7RH.

${ }^{2}$ Department of Medical Epidemiology and Biostatistics, Karolinska Institutet, Stocholm, Sweden. SE-171 77.

${ }^{3}$ King's College London, Medical School, Division of Cancer Studies, Section of Cancer Epidemiology, London, UK. SE1 9RT.

* - corresponding author.

Address: Department of Health Sciences, $2^{\text {nd }}$ Floor Adrian Building, University of Leicester. LE1 7RH. E-mail: mark.rutherford@le.ac.uk 


\section{Abstract:}

Background: Socioeconomic differences in cancer patient survival are known to exist for women diagnosed with breast cancer. Standard metrics tend not to place great emphasis on evaluating the actual impact of these differences.

Methods: We used two alternative, but related, methods of reporting the impact of socioeconomic differences for breast cancer patients in England and Wales. We calculated the average gain in life years for each patient should socioeconomic differences in relative survival be removed and show how this is related to the number of all-cause deaths that could be postponed by removing socioeconomic differences in cancer patient survival.

Results: Our results indicate that deprivation differences for women with breast cancer exist and result in women from more deprived areas losing a larger proportion of their life due to a diagnosis of cancer. We also estimate that on average 1.1 years could be gained for a 60 year old breast cancer patient in the most deprived group by improving their relative survival to match the least deprived group. However, our results also show that deprivation differences in general survival have a large impact on life expectancy; showing that over two-thirds of the gap in differential life expectancy is explained by differences in other-cause survival.

Conclusion: Socioeconomic differences in relative survival have an impact on life expectancy for patients and result in higher early mortality for more deprived patients. However, differences in general survival across socioeconomic groups explain a larger proportion of the deprivation gap in life expectancy for breast cancer patients.

Keywords: Socioeconomic differences; avoidable deaths; relative survival; life expectancy. 


\section{Understanding the impact of socioeconomic differences in breast cancer survival in England and Wales: avoidable deaths and potential gain in expectation of life}

\section{Introduction}

Relative survival estimates are commonly used for making comparisons across subgroups of cancer patients. Relative survival analysis is useful when making these comparisons as it allows for a fair comparison when background mortality varies between subgroups. However, estimates of relative survival are not easy to interpret and it can be difficult to translate the estimates into measures that help to understand the actual impact of a cancer diagnosis. Under certain assumptions [1], relative survival is a net probability estimate, i.e. a measure of survival where it is not possible to die of causes other than cancer, and therefore it is important to consider these estimates in combination with the competing mortality from other causes to give absolute measures of risk. It is also the case that relative survival estimates are usually only given at single points in follow-up time and therefore do not portray the changing risk as a function of time since diagnosis nor the overall risk over the lifetime.

A recent publication has argued that more relevant measures for patients should be favoured over the traditionally reported estimates of relative survival [2]. One approach has been to report the number of deaths that would be avoided by a given point in the future if the excess mortality rate of one group was also experienced by the second $[3,4,5,6,7,8]$. This measure is highly dependent upon follow-up time, but is often reported at a time single point, usually at 5-years after diagnosis. A second measure that has also recently been re-introduced is the difference in the number of life years in one group compared to another $[9,10]$.

The avoidable deaths estimates are calculated by altering the cancer-specific (or excess) mortality for a subgroup of patients. This will lead to completely avoiding a proportion of deaths due to cancer. However, these deaths will then be replaced by deaths due to other causes at some point in the future; no death is truly avoidable, only postponed. Postponing these deaths will also impact directly on the life expectancy for the cohort of cancer patients. The avoidable deaths statistic has been misinterpreted in a number of settings due to the lack of understanding surrounding how heavily this measure depends on time since diagnosis. The loss in expectation of life is an overall summary measure over the entire lifetime of the patients in the cohort and is therefore less prone to misinterpretation.

In this paper, we will analyse a cohort of breast cancer patients from England and Wales to explore the use of different measures of reporting differences in survival between socioeconomic groups. We will show how it is possible to quantify how much life can be gained by applying the cancer survival experience of the least deprived to all patients. We will also explore how this measure is related to the number of deaths that can be postponed by improving cancer-specific survival. 
The aim of the paper is to increase awareness of the use of loss in expectation of life as a measure, and to highlight how useful this can be in describing differences in cancer patient survival. A concurrent aim of this paper is to explore the impact of deprivation differences in cancer survival in breast cancer patients in England and Wales.

\section{Methods}

\section{Data}

We used National Cancer Registry Data for women with a new diagnosis of breast cancer (International Classification of Diseases-10 site code - C50) resident in England and Wales between 1999 and 2009, with follow up for mortality until the end of 2010. We restricted the analysis to those aged $30-99$ (this excludes $0.46 \%$ of the original sample size for analysis) so that the models are not overly influenced by sparse data in the tails of the age distribution. Patients were categorised into five socioeconomic status groups ( $1=$ least deprived, $5=$ =most deprived) using national quintiles of the income domain of the Index of Multiple Deprivation (IMD) score of the Lower Super Output Area of patients' residence at diagnosis [11]. Information on age-at-diagnosis and deprivation status was complete for all of the patients. We used exact age-at-diagnosis rather than categorising.

\section{Statistical Methods}

We used a period analysis [12] to provide up-to-date estimates of survival. We used a 5-year period window (2005-2009 inclusive) to ensure that the estimates were not overly influenced by short-term survival estimates that may be outdated whilst also maintaining sufficient data to fit a relatively complex model.

We used a modelling framework in order to estimate relative survival for the breast cancer patients. We fitted a flexible parametric excess mortality model $[13,14,15]$ that incorporated both socioeconomic status (as a categorical variable for the 5 levels of the quintile groups) and age-atdiagnosis. The effect of age-at-diagnosis was captured by using a flexible non-linear function. We also allowed an interaction term between the 5 levels of deprivation and a simplified function for the effect of age-at-diagnosis. The main effects of deprivation and the non-linear function for age-atdiagnosis were allowed to be time-dependent. We applied a further restriction to the model in terms of only allowing the effect of deprivation to be time-dependent for the first 15 years of followup. This restriction ensured that the long-term estimates of deprivation-specific survival follow the expected pattern rather than making what seemed unrealistic extrapolations. Further details of the model are given in the Appendix. We also assessed the sensitivity of the results to some of the assumptions that are made as part of the extrapolation. Details of the sensitivity analysis are given in the Appendix.

We use the approach described by Andersson et al. [10] in order to estimate the loss in expectation of life from a flexible parametric excess mortality model. In order to estimate the number of avoidable deaths and the loss in expectation of life we incorporated the background population mortality for the patients using general population mortality files stratified by age, deprivation group and calendar year [16]. In order to make the projected estimates for the entire lifetime, it is 
necessary to input future general population mortality rates. Here, we make the simple assumption that the rates observed in $\mathbf{2 0 1 1}$ were to continue into the future in order to give a full long-term estimate for patients diagnosed recently.

\section{Results}

Table 1 describes the cohort in terms of the numbers of patients diagnosed in deprivation categories across various age groups. These age-groups are purely used for the tabulation and the effect of age in the statistical models is modelled continuously. Table 1 indicates that deprivation group 5 (the least deprived group) is the smallest in terms of the proportion of the population. The total cohort comprise 366,692 women with breast cancer.

Figure 1 shows three aspects of life expectancy in relation to age at diagnosis, each plotted for the most and least deprived groups. Firstly, the figure shows an estimate of the mean years remaining under the assumption that the cohort of patients did not have breast cancer, using the appropriate mortality rates from the population mortality file (dashed lines). The expected mortality for those in the least deprived group is lower than for the most deprived group across almost the entire age range, resulting in the mean years of life remaining being consistently higher for all ages up to 95 . Secondly, the graph shows the average life years remaining given a diagnosis of cancer for the least and most deprived groups (solid lines). For example, for a 30 year old the mean years of life remaining without breast cancer in the least deprived group is 56 and the corresponding mean years remaining given a breast cancer diagnosis is 30 . Whereas for the most deprived patients, a 30 year old without cancer has a mean years of life remaining of around 50 , with those who have had a diagnosis of breast cancer have an average of 27 years of life remaining. The difference between these two values for each of the deprivation groups gives the loss in expectation of life as a function of age (dotted lines in Figure 1). For the entire age-range there is little difference between the estimates of the loss in expectation of life for the two deprivation groups. That is, both groups lose a very similar amount of life on average from their years remaining without breast cancer when compared to the mean years remaining following a diagnosis of cancer. However, the most deprived patients have fewer expected life years remaining on average, and so this loss has a larger proportional impact on their life expectancy (see Table 2). The average loss in expectation of life is higher for younger patients. These patients have more years to lose in the first place, and in the case of breast cancer, suffer from a moderately high excess mortality due to the disease.

The calculated values for age-at-diagnosis 60 given in Table 2 show that for the most deprived cohort of patients, the average number of years remaining based on not having a diagnosis of cancer would be 23.1 years. The corresponding value for the least deprived group is almost 4 years longer (26.9 years). This reflects the different general survival for the two populations. The average years remaining if diagnosed with breast cancer indicates the experience of the cohorts given they have had a diagnosis of cancer at age 60, estimated to 22.3 and 18.2 for the least and most deprived groups respectively. Any difference between deprivation group 1 and 5 reflect differences in both expected and relative survival. The loss in expectation of life is the difference in life expectancy with 
and without cancer, and is estimated to be 4.7 and 4.9 for the least and most deprived groups respectively.

It is possible to quantify the differences in cancer mortality by recalculating the loss in expectation of life assuming that the excess mortality of the least deprived group applied to all other deprivation groups. This increases the average years of life remaining from 18.2 years to 19.3 years for the most deprived group. The gap between deprivation group 1 and 5 reduces from 4.1 to 3 . This shows that the proportion of the deprivation gap in number of years remaining that is due to differences in relative (cancer) survival is only around $27 \%$ (see Table 3 ), with the remaining difference explained by differences in non-cancer mortality. Table 2 also reports the proportion of life lost for each of the five deprivation groups. Using the observed data, it is clear that there are substantial differences in the average proportion of life lost due to a diagnosis of cancer across deprivation groups. If deprivation differences in cancer survival were eliminated, these differences in the proportions are dramatically reduced. For instance, for a women diagnosed at age 60 , the proportion of life lost in deprivation group 5 is $21.1 \%$. This would be reduced to $16.1 \%$ if the deprivation differences in just cancer (relative) survival were eliminated.

The values for patients diagnosed at 80 given in the lower portion of Table 2 show that the years of life remaining based on the population mortality file is 10.6 years for the least deprived patients, and 9.2 years for the most deprived women. This reveals the deprivation differences in the expected survival in the general population. A similar pattern is seen in the observed years of life remaining, with the cancer patients on average estimated to live 7.6 years in the least deprived group compared to 6.2 years in the most deprived group. If the relative survival experience for all patients is matched to that of the least deprived patients, a difference in the years remaining still persists due to the remaining deprivation differences in the expected survival. As for those aged 60, the proportion of life years lost is much more similar across the deprivation groups when recalculating the estimates with the relative survival for all matched to that of the least deprived group.

Table 2 also shows that the estimates of 5 -year relative survival for those women aged 40 are slightly lower than for patients aged 60 across all deprivation groups. This is consistent with previous evidence that breast cancer in younger women has a greater relative impact on survival [6]. Table 2 also indicated that women aged 40 lose around 3 times as many years as those diagnosed at age 60 . This is partially to do with the increased relative survival and the long-term impact of continued excess mortality for breast cancer [17]. However, the main reason that women of younger ages lose a greater number of years is that they have more years to lose initially. Women aged 40 would be expected to live in excess of 40 years on average for all of the deprivation groups. This means that the relative impact on their life expectancy has a larger absolute effect.

Table 3 shows the deprivation gap, defined as the difference between the mean years of life remaining in all other deprivation groups compared to deprivation group 1, for ages 60 and 80 . The table also reports the corresponding deprivation gap if differences in cancer (relative) survival were eliminated. These estimates were used to calculate the proportion of the deprivation gap due to differences in relative (cancer) survival, with the remaining difference explained by differences in non-cancer mortality (see the $3^{\text {rd }}$ column of Table 3). For those diagnosed at age 80 in deprivation group $5,43 \%$ of the deprivation gap is explained by the difference in cancer survival. However, for 
younger ages and the other deprivation groups, the estimates tend to be below $30 \%$, with over twothirds of the difference in life expectancy being explained by other cause mortality differences.

Figure 2 (a) shows the total number of avoidable deaths as a function of follow-up time over all ages $(30+)$ for the 4 deprivation groups. These deaths are those that are avoided by applying the excess mortality of the least deprived group rather than the observed excess mortality in each group. These figures relate to the breast cancer cohort in England and Wales diagnosed in the year 2009. The total avoidable deaths reach a maximum at around 5 years after diagnosis. Nearly 600 deaths that would have occurred within the first 5 years given the deprivation differences in relative survival could have been postponed beyond this point if the differences in excess mortality across deprivation were eradicated. However, as follow-up increases the number of deaths that are postponed beyond a given point in follow-up decreases due to the balance between the competing causes of mortality. By 60 years of follow-up, the avoidable deaths estimate is very low because the vast majority of the cohort is projected to have died irrespective of the cancer-specific mortality that is applied to the group. Even if there was no excess mortality associated with a diagnosis of cancer, the cohort would eventually be depleted by other-causes of death.

Figure 2 (b) shows the avoidable deaths given in Figure 2 (a) stratified into four age groupings. The totals of these four graphs at each point in follow-up sum to the values given in Figure 2 (a). The contribution to the total avoidable deaths over all ages is smallest from the oldest age group, particularly in the long-term. Among older patients death cannot be "avoided" for as long, but they do contribute a substantial amount to the estimates in the short-term; the make-up of these figures depends on both the incidence of breast cancer by age and also the differences in relative survival at different ages. What is also clear from the bottom-right panel of Figure 2 (b) is that there are negative values calculated for the avoidable deaths in deprivation group 2 . This reflects the fact that for the very oldest breast cancer patients, those in deprivation group 2 appear to have higher relative survival estimates than those in deprivation group 1.

Table 4 shows the overall values for the cohort of breast cancer patients diagnosed in England and Wales in 2009. These values can be calculated analytically, but can also be achieved by calculating the area under (integrating) the avoidable deaths curves presented in Figure 2 (see the mathematical details in the Appendix). The first column indicates the total life years lost due to a cancer diagnosis in the five deprivation groups. The second column in the table shows the total life years that could have been saved by equalising the deprivation differences in relative survival. This indicates that around $6 \%$ of the total life years lost could have been prevented by removing relative survival differences across deprivation.

\section{Discussion}

Our results confirm that deprivation differences for women with breast cancer exist and result in women from more deprived areas losing a larger proportion of their life due to a diagnosis of cancer. An 80 year-old patient from the most deprived areas of England and Wales will lose around $33 \%$ of their remaining life expectancy following a cancer diagnosis, whereas an 80 year old from the least deprived area would lose around $28 \%$ of their remaining life. However, our results also show that deprivation differences in general survival have a larger impact on life expectancy. The deprivation 
gap in life years remaining is dominated by differences due to other causes rather than differences in cancer survival, with over two-thirds of the gap explained by differences in other causes for most patients.

Relative survival is a common approach for comparing net survival between subgroups of cancer patients and different populations. There are good reasons why some analyses and comparisons are suited to the use of relative survival. However, other methods should be considered when trying to give a fuller understanding of the impact of cancer. We are not suggesting that using avoidable deaths or loss in expectation of life to summarise differences should be approaches to replace the relative survival analysis, but they can be obtained as supplementary estimates from the very same models used to estimate relative survival. These measures have greater emphasis on interpretability; using units that are easier to understand and ensuring that other causes of death are appropriately incorporated. We have also shown how these two measures are directly related to each other - see Appendix for a mathematical justification.

Deprivation differences in survival exist for patients diagnosed with breast cancer and it is important to understand the reasons for these differences. In this paper, we have projected forwards across the whole lifetime to estimate the expectation of life for patients who have a diagnosis of breast cancer. This increases the understanding of how much of an impact deprivation differences, in terms of both relative and expected survival, have on women diagnosed across different ages. We have shown that by using extrapolations of key survival quantities we can give up-to-date estimates of life expectancy and the loss in life expectancy for women with breast cancer.

For the very oldest patients, the effect of deprivation is less strong, and in some cases reversed. This possibly reflects selection due to the fact that the people remaining in the most deprived category are healthy survivors and in the top proportion of their group in terms of survival. However, further work should be undertaken to fully understand the effect of deprivation in the very elderly and to try to understand the level of uncertainty for this group.

When using statistical models to project beyond the range of the data, it is important to consider the implications of extrapolations. We use a model-based approach, and make projections of the relative survival, rather than projecting the all-cause survival until it reaches zero. This has been shown to be a more effective way of reliably estimating the survival experience for a cohort of cancer patients [10]. We have made the assumption that beyond 15 years of follow-up, the effect of deprivation will be proportional and be the same across all age-groups. This assumption ensured that any long-term diminishing effects of deprivation and age did not lead to extrapolations which would have excess hazard ratios that decreased below 1 . We have conducted a sensitivity analysis to assess the impact of this assumption on the results (see Appendix). The sensitivity analysis indicates that the results are fairly insensitive to moderate differences in the point selected. In this application, we also make a simple assumption about the future expected rates for the general population; assuming the age, sex and deprivation-specific rates of mortality in 2011 will continue into the future to make the long-term estimates.

Loss in expectation of life and estimates of how many deaths can be postponed beyond a certain point in time give further understanding to the impact of deprivation differences in cancer patient survival. The loss in expectation of life can be used to understand how the differences impact across the whole lifetime for patients; this is particularly useful for breast cancer where patients are at a 
continued excess risk in the long-term following a diagnosis of cancer. These measures are also useful as they are in units that are more readily understandable than traditional approaches for expressing differences in survival. Although we agree that relative survival should be continued to be used in some analyses, it is also important to consider other metrics that may be more relevant when expressing differences between deprivation, and other population groups.

\section{Acknowledgements}

Mark J Rutherford is funded by a Cancer Research UK Postdoctoral Fellowship (CRUK_A13275). We would also like to acknowledge a useful discussion with Bernard Rachet over the differential effect of deprivation for the very oldest ages which contributed to our discussion point on the topic.

\section{Conflict of Interest}

The authors declare that they have no conflict of interest. 


\section{References}

[1] Perme MP, Stare J, Estève J. On estimation in relative survival. Biometrics. 2012 Mar;68(1):113-120. Available from: http://dx.doi.org/10.1111/j.1541-0420.2011.01640.x.

[2] Eloranta S, Adolfsson J, Lambert PC, Stattin P, Akre O, Andersson TML, et al. How can we make cancer survival statistics more useful for patients and clinicians: an illustration using localized prostate cancer in Sweden. Cancer Causes Control. 2013 Mar;24(3):505-515. Available from: http://dx.doi.org/10.1007/s10552-012-0141-5.

[3] Abdel-Rahman M, Stockton D, Rachet B, Hakulinen T, Coleman MP. What if cancer survival in Britain were the same as in Europe: how many deaths are avoidable? $\mathrm{Br} \mathrm{J}$ Cancer. 2009;101(S2):S115-S124. Available from: http://dx.doi.org/10.1038/sj.bjc.6605401.

[4] Rachet B, Ellis L, Maringe C, Chu T, Nur U, Quaresma M, et al. Socioeconomic inequalities in cancer survival in England after the NHS cancer plan. Br J Cancer. 2010 Aug;103(4):446-453. Available from: http://dx.doi.org/10.1038/sj.bjc.6605752.

[5] Pokhrel A, Martikainen P, Pukkala E, Rautalahti M, Seppa K, Hakulinen T. Education, survival and avoidable deaths in cancer patients in Finland. Br J Cancer. 2010 Sep;103(7):1109-1114. Available from: http://dx.doi.org/10.1038/sj.bjc.6605861.

[6] Lambert PC, Holmberg L, Sandin F, Bray F, Linklater KM, Purushotham A, et al. Quantifying differences in breast cancer survival between England and Norway. Cancer Epidemiol. 2011 Dec;35(6):526-533. Available from: http://dx.doi.org/10.1016/j.canep.2011.04.003.

[7] Ellis L, Coleman MP, Rachet B. How many deaths would be avoidable if socioeconomic inequalities in cancer survival in England were eliminated? A national population-based study, 1996-2006. European Journal of Cancer. 2012 Jan;48(2):270-278. Available from: http://www.sciencedirect.com/science/article/pii/S0959804911007957.

[8] Rutherford MJ, Hinchliffe SR, Abel GA, Lyratzopoulos G, Lambert PC, Greenberg DC. How much of the deprivation gap in cancer survival can be explained by variation in stage at diagnosis: An example from breast cancer in the East of England. Int J Cancer. 2013 Nov;133(9):2192-2200. Available from: http://dx.doi.org/10.1002/ijc.28221.

[9] Hakama M, Hakulinen T. Estimating the expectation of life in cancer survival studies with incomplete follow-up information. Journal of Chronic Diseases. 1977;30(9):585 - 597. Available from: http://www.sciencedirect.com/science/article/B7GH4-4COMW3Y-2VS/2/54bfdc7e1804433487cc4e731d16cc24. 
[10] Andersson TML, Dickman PW, Eloranta S, Lambe M, Lambert PC. Estimating the loss in expectation of life due to cancer using flexible parametric survival models. Stat Med. 2013 Aug;Available from: http://dx.doi.org/10.1002/sim.5943.

[11] Neighbourhood Renewal Unit. The English indices of deprivation 2004 (revised). Office for the Deputy Prime Minister, London (2004);.

[12] Brenner $\mathrm{H}$, Hakulinen T. Up-to-date long-term survival curves of patients with cancer by period analysis. Journal of Clinical Oncology. 2002;20(3):826-832. Available from: http://www.scopus.com/inward/record.url?eid=2-s2.0-

0036467651\&partnerID=40\&md5=53960e18b9cc93522280a10d5e5f3d02.

[13] Royston P, Parmar MKB. Flexible parametric proportional-hazards and proportionalodds models for censored survival data, with application to prognostic modelling and estimation of treatment effects. Statistics in Medicine. 2002;21(15):2175-2197.

[14] Nelson CP, Lambert PC, Squire IB, Jones DR. Flexible parametric models for relative survival, with application in coronary heart disease. Stat Med. 2007 Dec;26(30):5486-5498.

[15] Royston P, Lambert PC. Flexible Parametric Survival Analysis Using Stata: Beyond the Cox Model. StataCorp LP; 2011.

[16] Cancer Research UK Cancer Survival Group (2006). Life tables for cancer survival analysis;. Downloaded from www.Ishtm.ac.uk/ncde/cancersurvival/tools; Department of Non-Communicable Disease Epidemiology, London School of Hygiene \& Tropical Medicine, UK.

[17] Woods LM, Rachet B, Lambert PC, Coleman MP. 'Cure' from breast cancer among two populations of women followed for 23 years after diagnosis. Annals of Oncology. 2009;20(8):1331-1336. Available from: http://annonc.oxfordjournals.org/content/20/8/1331.abstract.

[18] Rutherford MJ, Crowther MJ, Lambert PC. The use of restricted cubic splines to approximate complex hazard functions in the analysis of time-to-event data: a simulation study. Journal of Statistical Computation and Simulation. 2013 Oct;p. 1-17. Available from: http://dx.doi.org/10.1080/00949655.2013.845890. 


\section{$\underline{\text { Statistical Appendix }}$}

\section{Model Specification}

The full details of the statistical model used are detailed below. We used the stpm 2 command for the statistical software package, Stata. We fitted a flexible parametric excess mortality model that incorporated both deprivation (as a categorical variable for the 5 levels of the quintile groups) and age-at-diagnosis. We use 5 degrees of freedom for the spline function to capture the shape of the log baseline cumulative excess hazard using the default knot locations at the centiles of the log of the event times. The effect of age-at-diagnosis was captured by using a flexible non-linear function (a restricted cubic spline function with 5 degrees of freedom, again the knots were placed at equally spaced centiles). We also allowed an interaction term between the 5 levels of deprivation and a simplified spline function for the effect of age-at-diagnosis ( 3 degrees of freedom). The main effects of deprivation and the spline functions for age-at-diagnosis were all allowed to be time-dependent through further spline functions to model the effect of time since diagnosis (a simplified spline function with 3 degrees of freedom and default knot locations). We applied a further restriction to the model in terms of only allowing the effect of deprivation to be time-dependent for the first 15 years of follow-up. This restriction ensured that the long-term estimates of deprivation-specific survival follow the expected pattern rather than making what seemed unrealistic extrapolations.

\section{$\underline{\text { Sensitivity to assumptions }}$}

In making the extrapolations, it was necessary to make assumptions about the continuation of the relative survival curve into the long-term, particularly for younger patients at diagnosis. We applied a constraint on the long-term estimates (greater than 15 years after diagnosis) to enforce a proportional effect of deprivation. Here we show the equivalent estimates to Table 4 under a number of different assumptions to show the sensitivity of the results. The results are given for 2 alternative scenarios in Table A1 and compared to those given in Table 4. The results show that the choice of the point from which to make the constraint does not have a huge impact on the overall estimates. The choice and number of knots for flexible parametric models can also be important. However, it has been shown that provided sufficient complexity is allowed, the approach performs well in simulations [18]. 


\begin{tabular}{|c|c|c|c|c|}
\hline $\begin{array}{l}\text { Scenarios for } \\
\text { comparison }\end{array}$ & & $\begin{array}{c}\text { Group size in } \\
2009\end{array}$ & Total Years Lost & $\begin{array}{l}\text { Total years available to } \\
\text { gain if relative survival as } \\
\text { in deprivation group } 1\end{array}$ \\
\hline \multirow{6}{*}{$\begin{array}{l}\text { Proportional } \\
\text { beyond } \\
12 \text { years }\end{array}$} & Dep group 1 & 8,805 & $52,011.39$ & 0 \\
\hline & Dep Group 2 & 8,993 & $50,469.34$ & $1,197.27$ \\
\hline & Dep group 3 & 8,208 & $45,353.25$ & $2,611.83$ \\
\hline & Dep group 4 & 7,228 & $35,734.73$ & $4,062.57$ \\
\hline & Dep group 5 & 5,935 & $52,011.39$ & $5,911.44$ \\
\hline & Total & 39,169 & $224,142.9$ & $13,783.1$ \\
\hline \multirow{6}{*}{$\begin{array}{l}\text { Proportional } \\
\text { beyond } \\
15 \text { years } \\
\text { (same as } \\
\text { Table 4) }\end{array}$} & Dep group 1 & 8,805 & $51,951.07$ & 0 \\
\hline & Dep Group 2 & 8,993 & $50,557.73$ & $1,297.40$ \\
\hline & Dep group 3 & 8,208 & $45,454.81$ & $2,708.76$ \\
\hline & Dep group 4 & 7,228 & $40,627.54$ & $4,146.93$ \\
\hline & Dep group 5 & 5,935 & $35,801.14$ & $6,001.33$ \\
\hline & Total & 39,169 & 224392.30 & 14154.41 \\
\hline \multirow{6}{*}{$\begin{array}{l}\text { Proportional } \\
\text { beyond } \\
18 \text { years }\end{array}$} & Dep group 1 & 8,805 & $52,018.71$ & 0 \\
\hline & Dep Group 2 & 8,993 & $50,590.84$ & $1,339.60$ \\
\hline & Dep group 3 & 8,208 & $45,539.26$ & $2,752.95$ \\
\hline & Dep group 4 & 7,228 & $40,707.27$ & $4,181.56$ \\
\hline & Dep group 5 & 5,935 & $35,831.30$ & $5,990.06$ \\
\hline & Total & 39,169 & $22,4687.4$ & $14,264.17$ \\
\hline
\end{tabular}

Table A1: Table showing the lack of sensitivity to the assumption of proportionality made in the extrapolations for the effect of deprivation.

Equivalence of gain in life expectancy and cumulative avoidable deaths

We used a model to estimate relative survival, $\mathrm{R}_{i}(\mathrm{t})$, for each individual, $i$, which can be formulated as:

$\mathrm{R}_{i}(\mathrm{t})=\frac{S_{i}(t)}{S_{i}^{*}(t)^{\prime}}$

where $S_{i}(t)$ is the all-cause survival function in the cohort, and $S_{i}^{*}(t)$ is the corresponding expected survival obtained from the appropriate population mortality file estimates.

The number of expected deaths at time, $\mathrm{t}$, in the general population for individuals with $\mathrm{N}$ individuals with the same estimated survival can be estimated by:

$D^{*}(t)=N\left(1-S_{i}^{*}(t)\right)$.

The number of all-cause deaths at time, $t$, in the general population for the same subcohort can be estimated by:

$D(t)=N\left(1-S_{i}^{*}(t) \mathrm{R}_{i}(\mathrm{t})\right)$. 
Consider the situation where we have two groups for comparison. Let $R_{1}(t)$ be the relative survival for one group and, $R_{2}(t)$ for the second. With $R_{1}(t)>R_{2}(t)$.

The avoidable deaths for group 2 can be estimated by:

$$
\begin{aligned}
A D(t) & =N\left(1-R_{2}(t) S_{2}^{*}(t)\right)-N\left(1-R_{1}(t) S_{2}^{*}(t)\right) \\
& =N\left(R_{1}(t)-R_{2}(t)\right) S_{2}^{*}(t)
\end{aligned}
$$

For the same two groups we can also formulate the gain in the expectation of life. This would be the gain in life years that could be made by adopting the relative survival experience of group 1 for group 2.

$$
\begin{aligned}
G i E & =\left(\int_{0}^{\infty} S_{2}^{*}(t) d t-\int_{0}^{\infty} R_{2}(u) S_{2}^{*}(t) d t\right)-\left(\int_{0}^{\infty} S_{2}^{*}(t) d t-\int_{0}^{\infty} R_{1}(u) S_{2}^{*}(t) d t\right) \\
& \left.=\int_{0}^{\infty}\left(R_{1}(u)-R_{2}(u)\right) S_{2}^{*}(t) d t\right) .
\end{aligned}
$$

This can then be multiplied by $\mathrm{N}$ to give an overall measure rather than the expectation. This shows that integrating the avoidable death curve over $t$ will result in an estimate of the difference in the loss in expectation of life between the two groups. 


\section{Figures:}

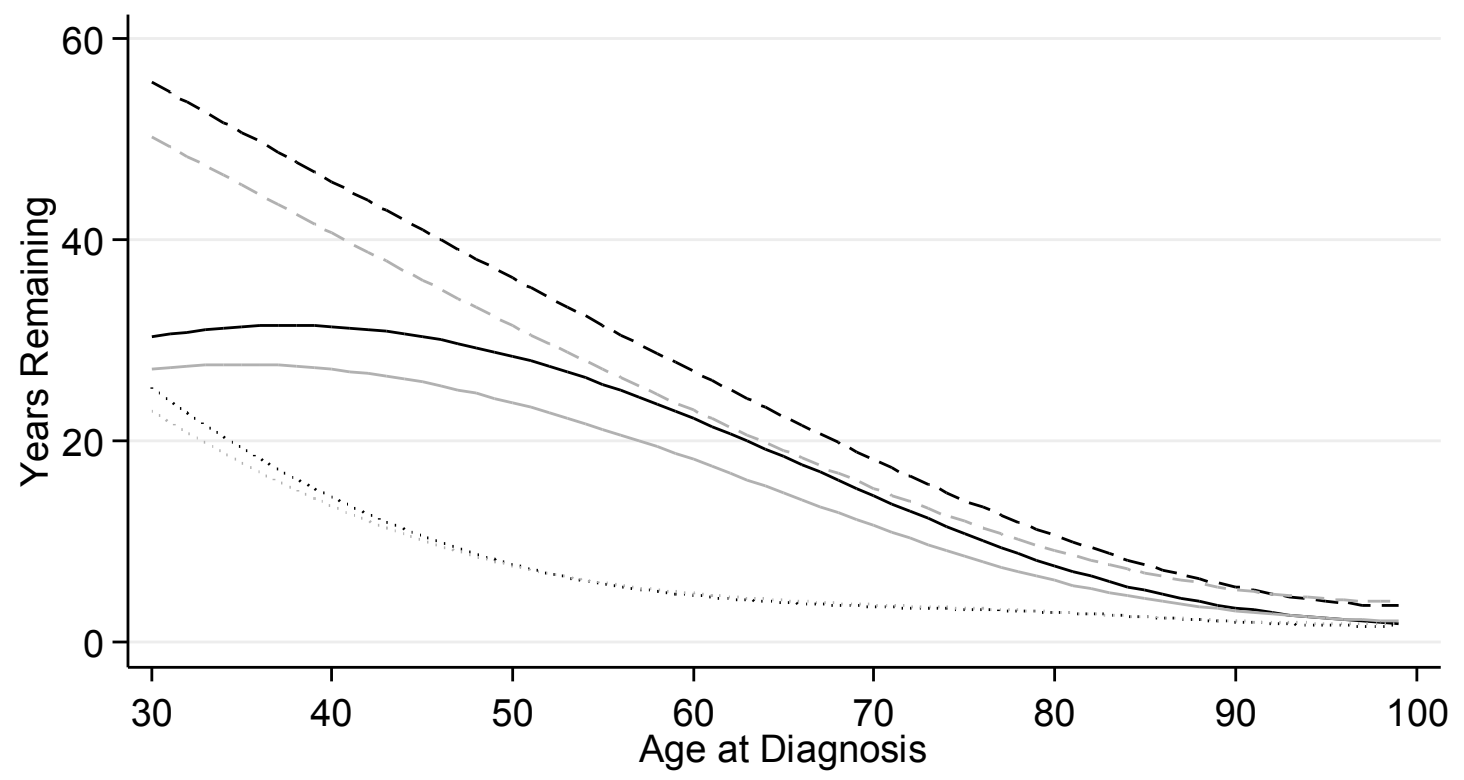

Mean Years Remaining:

Without Cancer

- Least Deprived

Most Deprived

With Cancer

- - - Least Deprived

Most Deprived

Loss in Expectation of Life:

Least Deprived

Most Deprived

Figure 1: Mean expected years remaining (without breast cancer), Mean observed years remaining (with cancer), and loss in expectation of life as functions of age. 
(a)

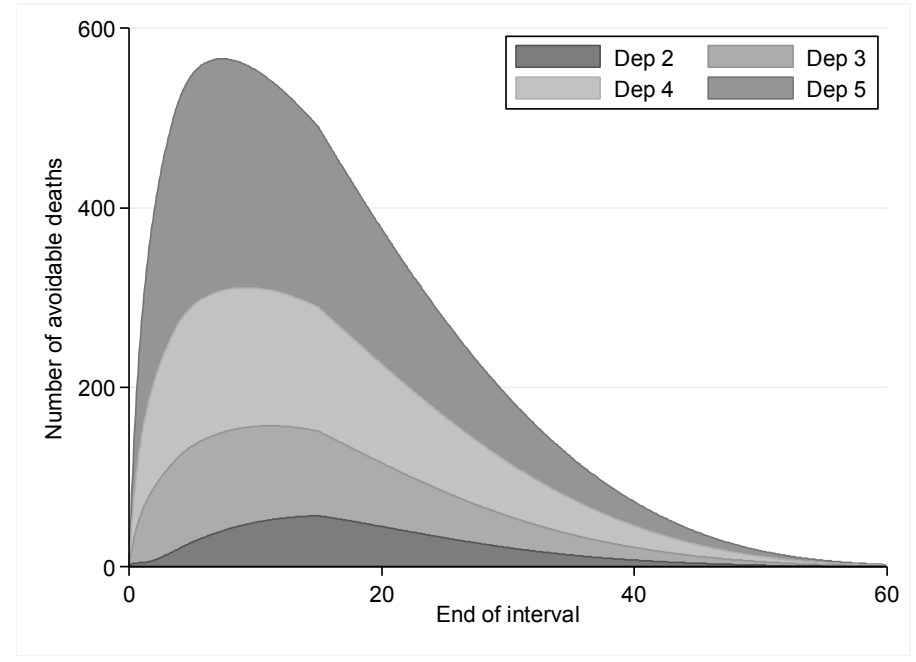

(b)
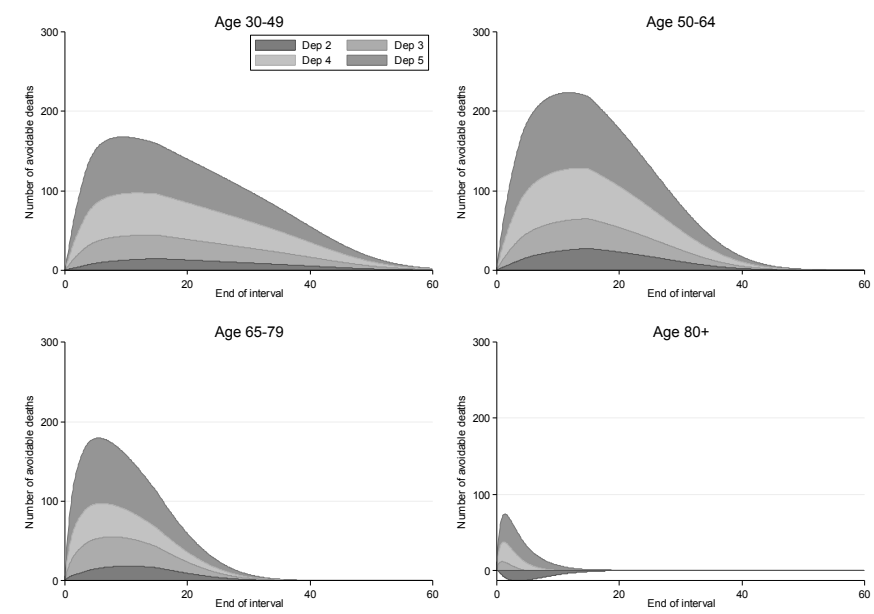

Figure 2: (a) Long-term projections of the avoidable deaths for patients diagnosed in a 2009 partitioned by deprivation (the groups are stacked to give the overall measure). (b) The equivalent estimates for 2009 stratified by 4 age-groups. 


\section{Tables:}

Table 1: Number of patients in age and deprivation categories for the entire cohort (diagnosis window (1999-2009).

\begin{tabular}{r|rrr|r}
\hline & \multicolumn{3}{|c|}{ Age Group } & $\mathbf{7 5 +}$ \\
\hline Deprivation Group & $\mathbf{3 0 - 5 4}$ & $\mathbf{5 5 - 7 4}$ & 14088 & Total \\
\hline $\mathbf{1}$ & 29383 & 39448 & 16694 & 82919 \\
$\mathbf{2}$ & 27282 & 39132 & 17093 & 77551 \\
$\mathbf{3}$ & 24765 & 35693 & 15723 & 68450 \\
$\mathbf{4}$ & 22227 & 30500 & 11746 & 54664 \\
\hline $\mathbf{5}$ & 18855 & 24063 & $\mathbf{7 5 3 4 4}$ & 366692 \\
\hline Total & 122512 & 168836 & &
\end{tabular}


Table 2: Estimates for 40, 60 and 80 year olds for the average values of lifetime remaining (years).

\begin{tabular}{|c|c|c|c|c|c|c|c|c|}
\hline \multirow[b]{2}{*}{ Age-at-diagnosis: 40} & \multicolumn{5}{|c|}{ Using observed excess mortality } & \multicolumn{3}{|c|}{ Using the excess mortality of deprivation group 1} \\
\hline & $\begin{array}{l}\text { 5-year } \\
\text { relative } \\
\text { survival } \\
(\%)\end{array}$ & $\begin{array}{l}\text { Mean years } \\
\text { remaining } \\
\text { without breast } \\
\text { cancer }\end{array}$ & $\begin{array}{l}\text { Mean years } \\
\text { remaining with } \\
\text { breast cancer }\end{array}$ & $\begin{array}{l}\text { Mean loss in life } \\
\text { expectancy due } \\
\text { to breast cancer }\end{array}$ & \begin{tabular}{|l|} 
Percentage of \\
life lost due to \\
breast cancer (\%)
\end{tabular} & $\begin{array}{l}\text { Mean years } \\
\text { remaining with } \\
\text { breast cancer }\end{array}$ & $\begin{array}{l}\text { Mean loss in } \\
\text { life expectancy } \\
\text { due to breast } \\
\text { cancer }\end{array}$ & $\begin{array}{l}\text { Percentage of life } \\
\text { lost due to breast } \\
\text { cancer (\%) }\end{array}$ \\
\hline Deprivation group 1 (least) & 87.9 & 45.9 & 31.4 & 14.4 & 31.5 & 31.4 & 14.4 & 31.4 \\
\hline Deprivation group 2 & 87.5 & 44.7 & 30.6 & 14.1 & 31.6 & 30.8 & 13.9 & 31.1 \\
\hline Deprivation group 3 & 86.6 & 43.8 & 29.8 & 14.1 & 32.1 & 30.3 & 13.5 & 30.8 \\
\hline Deprivation group 4 & 85.3 & 42.5 & 28.5 & 13.8 & 32.9 & 29.6 & 12.9 & 30.4 \\
\hline Deprivation group 5 (most) & 83.9 & 40.7 & 27.2 & 13.5 & 33.2 & 28.5 & 12.2 & 29.9 \\
\hline \multicolumn{9}{|l|}{ Age-at-diagnosis: 60} \\
\hline Deprivation group 1 (least) & 91.4 & 26.9 & 22.3 & 4.7 & 17.4 & 22.3 & 4.7 & 17.4 \\
\hline Deprivation group 2 & 90.8 & 26.0 & 21.3 & 4.6 & 17.9 & 21.5 & 4.4 & 17.0 \\
\hline Deprivation group 3 & 90.2 & 25.2 & 20.6 & 4.6 & 18.2 & 21.0 & 4.2 & 16.8 \\
\hline Deprivation group 4 & 89.0 & 24.3 & 19.6 & 4.7 & 19.2 & 20.2 & 4.0 & 16.5 \\
\hline Deprivation group 5 (most) & 86.8 & 23.1 & 18.2 & 4.9 & 21.2 & 19.3 & 3.7 & 16.2 \\
\hline \multicolumn{9}{|l|}{ Age-at-diagnosis: 80} \\
\hline Deprivation group 1 (least) & 74.9 & 10.6 & 7.6 & 3.0 & 28.2 & 7.6 & 3.0 & 28.2 \\
\hline Deprivation group 2 & 74.9 & 10.0 & 7.3 & 2.7 & 27.2 & 7.2 & 2.7 & 27.3 \\
\hline Deprivation group 3 & 72.5 & 9.7 & 6.9 & 2.8 & 29.3 & 7.1 & 2.6 & 27.1 \\
\hline Deprivation group 4 & 72.2 & 9.4 & 6.7 & 2.7 & 29.1 & 6.9 & 2.5 & 26.9 \\
\hline Deprivation group 5 (most) & 67.5 & 9.2 & 6.2 & 3.1 & 33.1 & 6.7 & 2.5 & 26.9 \\
\hline
\end{tabular}


Table 3: Proportion of deprivation gap due to differences in relative survival. Deprivation gap calculated by comparing to deprivation group 1 .

\begin{tabular}{|c|c|c|c|}
\hline \multicolumn{4}{|c|}{ Age-at-diagnosis: 40} \\
\hline Deprivation Group & $\begin{array}{l}\text { Deprivation gap in } \\
\text { mean years } \\
\text { remaining with } \\
\text { breast cancer } \\
\text { (years) }\end{array}$ & $\begin{array}{l}\text { Deprivation gap in } \\
\text { mean years } \\
\text { remaining with } \\
\text { breast cancer if RS } \\
\text { of dep } 1 \text { applied to } \\
\text { all (years) }\end{array}$ & $\begin{array}{l}\text { Percentage of } \\
\text { deprivation gap } \\
\text { due to cancer } \\
\text { (relative) survival } \\
\text { differences }\end{array}$ \\
\hline 2 & 0.8 & 0.6 & $25.4 \%$ \\
\hline 3 & 1.7 & 1.1 & $34.0 \%$ \\
\hline 4 & 2.9 & 1.9 & $35.8 \%$ \\
\hline 5 & 4.2 & 2.9 & $31.8 \%$ \\
\hline \multicolumn{4}{|c|}{ Age-at-diagnosis: 60} \\
\hline Deprivation Group & $\begin{array}{l}\text { Deprivation gap in } \\
\text { mean years } \\
\text { remaining with } \\
\text { breast cancer } \\
\text { (years) }\end{array}$ & $\begin{array}{l}\text { Deprivation gap in } \\
\text { mean years } \\
\text { remaining with } \\
\text { breast cancer if RS } \\
\text { of dep } 1 \text { applied to } \\
\text { all (years) }\end{array}$ & $\begin{array}{l}\text { Percentage of } \\
\text { deprivation gap } \\
\text { due to cancer } \\
\text { (relative) survival } \\
\text { differences }\end{array}$ \\
\hline 2 & 0.9 & 0.7 & $23.3 \%$ \\
\hline 3 & 1.6 & 1.3 & $21.6 \%$ \\
\hline 4 & 2.7 & 2.0 & $24.3 \%$ \\
\hline 5 & 4.1 & 2.9 & $28.1 \%$ \\
\hline \multicolumn{4}{|c|}{ Age-at-diagnosis: 80} \\
\hline Deprivation Group & $\begin{array}{l}\text { Deprivation gap in } \\
\text { mean years } \\
\text { remaining with } \\
\text { breast cancer } \\
\text { (years) }\end{array}$ & $\begin{array}{l}\text { Deprivation gap in } \\
\text { mean years } \\
\text { remaining with } \\
\text { breast cancer if RS } \\
\text { of dep } 1 \text { applied to } \\
\text { all (years) }\end{array}$ & $\begin{array}{l}\text { Percentage of } \\
\text { deprivation gap } \\
\text { due to cancer } \\
\text { (relative) survival } \\
\text { differences }\end{array}$ \\
\hline 2 & 0.4 & 0.4 & $0.0 \%$ \\
\hline 3 & 0.7 & 0.5 & $27.5 \%$ \\
\hline 4 & 0.9 & 0.7 & $22.6 \%$ \\
\hline 5 & 1.5 & 0.9 & $39.4 \%$ \\
\hline
\end{tabular}


Table 4: Total life years lost in the cohort, and the years lost over the cohort due to relative survival differences in deprivation.

\begin{tabular}{|c|c|c|c|}
\hline & $\begin{array}{c}\text { Group size in } \\
2009\end{array}$ & Total Years Lost & $\begin{array}{l}\text { Total years available to } \\
\text { gain if relative survival as } \\
\text { in deprivation group } 1\end{array}$ \\
\hline Dep group 1 & 8,805 & $51,951.07$ & $\begin{array}{ll} & 0\end{array}$ \\
\hline Dep Group 2 & 8,993 & $50,557.73$ & $1,297.40$ \\
\hline Dep group 3 & 8,208 & $45,454.81$ & $2,708.76$ \\
\hline Dep group 4 & 7,228 & $40,627.54$ & $4,146.93$ \\
\hline Dep group 5 & 5,935 & $35,801.14$ & $6,001.33$ \\
\hline Total & 39,169 & $224,392.30$ & $14,154.41$ \\
\hline
\end{tabular}

